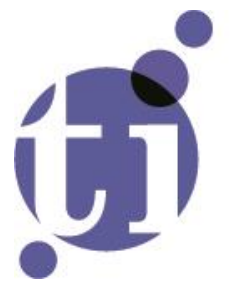

\title{
Common pool resources: Is there support for conservationists?
}

\section{Erik Ansink ${ }^{1}$}

Hans-Peter Weikard²

\footnotetext{
${ }^{1}$ Tinbergen Institute, Vrije Universiteit Amsterdam, the Netherlands

2 Wageningen University, the Netherlands
} 
Tinbergen Institute is the graduate school and research institute in economics of Erasmus University Rotterdam, the University of Amsterdam and VU University Amsterdam.

Contact: discussionpapers@tinbergen.nl

More TI discussion papers can be downloaded at http://www.tinbergen.nl

Tinbergen Institute has two locations:

Tinbergen Institute Amsterdam

Gustav Mahlerplein 117

1082 MS Amsterdam

The Netherlands

Tel.: +31(0)205984580

Tinbergen Institute Rotterdam

Burg. Oudlaan 50

3062 PA Rotterdam

The Netherlands

Tel.: +31(0)10408 8900 


\title{
Common pool resources: Is there support for conservationists?*
}

\author{
Erik Ansink ${ }^{\dagger} \quad$ Hans-Peter Weikard
}

November 1, 2018

\begin{abstract}
We examine the role of support for coalition stability in common pool resource games such as fisheries games. Some players may not want to join a coalition that jointly manages a resource. Still, because they benefit from spillovers, they may want to support the coalition with a transfer payment in order to set incentives for others to join. We find that the impact of support on equilibria of this game is limited to games with three or five players.
\end{abstract}

Keywords: Cartel games, Coalition formation; Common Pool Resources; Support

JEL classification: C72; D02; Q20

*We thank Michèle Breton, Harold Houba, Matthew McGinty, Maarten Punt, and seminar participants at EAERE 2017 and BioEcon 2017 for comments on an earlier version of this paper.

${ }^{\dagger}$ Department of Spatial Economics, Vrije Universiteit Amsterdam and Tinbergen Institute.

${ }^{\ddagger}$ Department of Social Sciences, Wageningen University. 


\section{Introduction}

Common pool resource (CPR) games are an important class of games that help to shed light on a diverse set of collective decision problems such as water extraction, rangeland management and fisheries policies. This paper explores the impact of support in a CPR game with coalition formation. The type of support we consider is a payment from singleton players to a coalition of players who coordinate their extraction efforts to jointly manage the resource. This type of support differs from earlier papers that have considered support in CPR games. The way we model support does not require an outside agency as in Ansink and Bouma (2013). 1 It also does not require commitment as in Long and Flaaten (2011) who consider supporting transfers by the coalition members to compensate singletons for joining the coalition. Rather, we model support in a sequential game following Ansink et al. (2018). They adapt the game setup of Carraro and Siniscalco (1993) to show that the impact of supporting transfers by singletons on coalition formation in public goods games may not require commitment.

While coalitions in public goods games are known to achieve a modest degree of cooperation (Hoel 1992; Carraro and Siniscalco 1993; Barrett 1994), a well-known result in the fisheries literature is that coalitions in CPR games are generally unstable when there are more than two players (Pintassilgo and Lindroos 2008). Larger coalitions can only be achieved when coalition membership brings about additional advantages such as Stackelberg leadership (Kwon 2006; Long and Flaaten 2011) or economies of scale (Johannesen and Skonhoft 2009). 22 This negative result on cooperation in the commons is driven by strong free-rider incentives with singleton players increasing extraction efforts in response to the coalition's resource conservation measures. Hence, it is worthwhile to assess whether the option of support can improve this result. We approach this option without requiring aspects like commitment, an outside agency, or additional advantages of coalition membership.

We model support using a sequential game that extends the standard two-stage coalition formation game. The extension is adding two stages at the start of the game in which the players choose whether to become a supporter in stage 1, and

\footnotetext{
${ }^{1}$ See also Zavalloni et al. (2018) for an alternative model setup.

${ }^{2}$ There are many alternative approaches to model cooperation in the commons, including e.g. Heintzelman et al. (2009) who model a partnership solution, Doyen and Péreau (2012) who use a viability approach and Polasky et al. (2006) who derive a folk theorem result for dynamic commons games.
} 
subsequently the set of supporters choose the level of a supporting transfer payment to the coalition in stage 2 . In the remaining two stages, a subset of the remaining players forms the coalition in stage 3 and all players choose their effort level in a standard CPR game in stage 4. Since the supporting transfer is shared by all coalition members, the purpose of the supporting transfer is to make coalition membership more attractive. For sufficiently high transfers, the incentive for coalition members to leave the coalition is removed completely. The question is, however, whether supporters are willing to pay sufficiently high transfers. We will see that the answer to this question is not always negative.

The main result of this paper is that there exist equilibrium solutions with positive levels of support. There exist only two such solutions, however. One equilibrium occurs when there are three players in the game, one of which is a supporter and the other two are coalition members. The other equilibrium occurs when there are five players in the game, such that two are supporters and three are coalition members. In larger games there are no stable coalitions. At first sight this does not seem to be good news for cooperation. Still, the result may be relevant as it covers the many situations where the number of players is indeed small. For all cases with four, six or a larger number of players we must draw the conclusion that support is not effective.

In the remainder of this paper, we introduce the model in Section 2 and present our main result in Section 3. Next, we illustrate this result in Section 4 and conclude in Section 5 .

\section{Support in a coalition game}

Consider a set $N=\{1,2, \ldots, n\}$ consisting of $n$ symmetric players. Our game is based on the standard two-stage coalition formation game (cf. Barrett 1994). We apply this game to a CPR following Pintassilgo and Lindroos (2008) and we extend it with support in line with Ansink et al. (2018). The resulting sequential game has four stages. We first introduce the stages in detail and subsequently solve the game backwards. 


\subsection{The four-stage game}

In stage 1, all players simultaneously decide whether or not to become a supporter. We denote this decision for each individual player by $\sigma_{i} \in\{0,1\} \forall i \in N$. When $\sigma_{i}=1$, player $i$ becomes a supporter and otherwise not. The outcome of stage 1 is a set $S=\left\{i: \sigma_{i}=1\right\}$, which comprises all supporters. Since players are symmetric we will often refer to the set of supporters by its size $s=|S|$.

In stage 2 , the set of supporters jointly decides on the level of the supporting transfer payment $t(s)$ to the coalition, with each supporter paying $t(s) / s$ due to (ex ante) symmetry. Since support precedes stage 3 coalition formation and stage 4 effort in the CPR game, supporters take into account the effect of their support level on these subsequent actions.

In stage 3 , given $t(s)$, the remaining $n-s$ players simultaneously decide whether or not to join the coalition. We denote this decision for each individual player by $\mu_{i} \in\{0,1\} \forall i \in N$. When $\mu_{i}=1$, player $i$ becomes a coalition member and otherwise not. The outcome of stage 3 is a set $M=\left\{i: \mu_{i}=1\right\}$, which comprises all members. At the end of stage 3 the set of players is partitioned into three subsets, $S, M$ and $F$, where the set $F=N \backslash\{S \cup M\}$ comprises the free-riders. Similar to our notation $s$ for the number of supporters, we will often refer to the coalition by its size $m=|M|$ and the set of free-riders by its size $f=|F|$.

In stage 4 , given the partitioning in player types $(s, m, f)$, all players simultaneously choose their individual effort level $e_{i}$ in the CPR game. Free-riders and supporters choose their effort level to maximize their individual payoffs while coalition members coordinate their effort levels to maximize joint payoffs.

Payoffs are determined using the canonical version of a CPR game (cf. MestertonGibbons 1993). In this game, we denote a players' individual harvest levels by $h_{i} \equiv q e_{i} X$, where $q$ is the so-called catchability coefficient, $e_{i}$ is individual effort and $X$ is the resource stock. The stock grows with intrinsic growth rate $r$ according to a logistic growth function $\frac{d X}{d t}=r X\left(1-\frac{X}{k}\right)$, where $k$ is the system's carrying capacity. The analysis is conducted for steady states where harvest equals growth such that $\sum_{i \in N} q e_{i} X=r X\left(1-\frac{X}{k}\right)$. Individual payoff is given by $v_{i}=p h_{i}-c e_{i}$, where $p$ is the price for selling one's harvest and $c$ is the marginal effort cost. With support, these payoffs are adjusted by transfers, paid by the supporters to the coalition members. As it is standard, a detailed description of this CPR game is relegated to the Appendix. 


\subsection{Solving the game}

We apply SPNE and solve the game backwards. The main difference with Ansink et al. (2018) is that we use a CPR game rather than a public goods game, which primarily implies different actions by all players in stage 4 . In this stage, equilibrium effort levels are determined from the best response functions of coalition members and singletons (i.e. supporters and free-riders). In the version of the CPR game that we consider here, the coalition - internalizing within-coalition externalities - will behave as a single player (Pintassilgo and Lindroos 2008). As a result, if $m$ players form a coalition, we have $n-m+1$ players harvesting the resource. Solving the system of best responses, equilibrium singleton payoffs $\lambda(m)$, excluding transfers, are defined as follows (a full derivation of payoffs is provided in the Appendix):

$$
\lambda(m)=\frac{r p k(1-\beta)^{2}}{(n-m+2)^{2}} \quad \forall i \in N \backslash M,
$$

where $\beta \equiv \frac{c}{p q k}$, such that the numerator consists of bio-economic model parameters only. Clearly, payoffs decrease in the number of players $n$ while they increase in the size of the coalition $m$. Since the coalition behaves as a single player, payoffs to members $\kappa(m)$, excluding transfers, are a fraction $\frac{1}{m}$ smaller:

$$
\kappa(m)=\frac{r p k(1-\beta)^{2}}{m(n-m+2)^{2}} \quad \forall i \in M .
$$

Equilibrium payoffs in the CPR game given by (1) and (2) can be used to spell out the stage 3 equilibrium conditions. Recall that in stage 3, all players that are not supporters choose whether or not to join the coalition. The equilibrium conditions for this stage are the standard internal and external stability conditions for cartel games due to D'Aspremont et al. (1983), which correspond to a Nash equilibrium in membership choices:

$$
\begin{aligned}
\kappa(m)+\frac{t}{m} & \geq \lambda(m-1), \\
\lambda(m) & >\kappa(m+1)+\frac{t}{m+1} .
\end{aligned}
$$

Internal stability implies that no coalition member has an incentive to leave the coalition, while external stability implies that no singleton has an incentive to join the coalition. Following Weikard (2009), we use a strict inequality sign in the external 
stability condition which simplifies the analysis in Section 3 below. The interpretation of this strict inequality is that a singleton who happens to be indifferent between staying a singleton or joining the coalition, is assumed to join. Condition (3) reveals that the main purpose of a supporting transfer $t \geq 0$ is to achieve internal stability for a coalition that would not be internally stable without the transfer. The related drawback is that the supporting transfer undermines external stability as can be seen from (4).

In stage 2 , supporters choose the transfer $t \geq 0$ with the objective of maximizing their joint payoff. This possibility of payoff-maximizing support was briefly considered in an extension by Ansink et al. (2018, Section 5.1) but it turned out not to be very effective in a public goods game.$^{3}$ In our CPR game, supporters choose a transfer payment as follows:

$$
t(s)=\arg \max _{t^{\prime}}\left(s \lambda\left(m\left(t^{\prime}\right)\right)-t^{\prime}\right)
$$

With slight abuse of notation, we denote by $m(t)$ the set of internally and externally stable coalitions under transfer $t$, as dictated by conditions (3) and (4). Below we will verify that $m(t)$ is unique for each given $t$. By selecting their transfer $t(s)$, the set of supporters $s$ is implicitly selecting the (internally and externally stable) coalition size $m$ that maximizes their payoffs $\lambda(m)-t(s) / s$. Note that the (implicit) selection of a coalition size which is not internally and externally stable could never constitute an equilibrium outcome of the game.

For stage 1, again we spell out equilibrium conditions, quite similar to those for stage 3. Recall that in stage 1, all players choose whether or not to become a supporter. The equilibrium conditions for this stage are similar to those used in Ansink et al. (2018), although we substitute $\lambda(m) / m$ for $\kappa(m)$ using (1) and (2). In interpreting these conditions, presented below, consider the following. In the sub-game following stage 1 , any player that is not a supporter will either become a coalition member or a free-rider in stage 3. A Nash equilibrium in stage 1 decisions implies that no supporter deviates from $\sigma_{i}=1$ and no other player deviates from $\sigma_{i}=0$, taking into account these subsequent stage 3 decisions. It follows that we

\footnotetext{
${ }^{3}$ In Section $\sqrt{5}$ we discuss a more effective model alternative.
} 
have four stability conditions in stage 1 of the game:

$$
\begin{aligned}
\lambda(m(t(s)))-\frac{t(s)}{s} & \geq \frac{\lambda(m(t(s-1)))+t(s-1)}{m(t(s-1))}, \\
\lambda(m(t(s)))-\frac{t(s)}{s} & \geq \lambda(m(t(s-1))), \\
\lambda(m(t(s))) & \geq \lambda(m(t(s+1)))-\frac{t(s+1)}{s+1}, \\
\frac{\lambda(m(t(s)))+t(s)}{m(t(s))} & \geq \lambda(m(t(s+1)))-\frac{t(s+1)}{s+1} .
\end{aligned}
$$

Conditions (6a) and (6b) are two internal stability conditions for the set of supporters $S$. They state that no supporter would deviate in stage 1 to become a member or free-rider in stage 3. Likewise, conditions (6c) and (6d) are two external stability conditions for the set of supporters $S$. They state that no singleton (who would choose to become a free-rider or a member in stage 3) would deviate to become a supporter in stage 1.

Summarizing, the game is solved by checking the equilibrium conditions for stage 1 and stage 3, given an optimal choice of the supporting transfer by the supporters in stage 2 and optimal effort choices by all players in stage 4.

\section{Equilibria with support}

In Section 2.2 we have derived six conditions for the existence of an equilibrium with supporters. Such equilibria can be identified by the numbers of supporters and members as established in stages 1 and 3 of the game, for some given number of players $n$. We proceed to check whether there exist such combinations $(s, m, f)$ that satisfy all six equilibrium conditions (3), (4), and (6a)- (6d). The answer is yes, there exist two equilibrium solutions with support for, respectively, $n=3$ and $n=5$ : $\left(s^{*}, m^{*}, f^{*}\right)=(1,2,0)$ and $\left(s^{*}, m^{*}, f^{*}\right)=(2,3,0)$. Deriving these equilibria is rather tedious. This will become clear in the remainder of this section, which serves as a proof of this result, while in Section 4 we provide an example to illustrate.

Given stage 4 optimal harvest levels and associated payoffs, we start with the stage 3 stability conditions (3) and (4). We first verify the existence of at least one combination of integers $(m, n)$ with $1<m \leq n$ such that these conditions hold.$^{4}$

\footnotetext{
${ }^{4}$ Note that a similar analysis was done by Ansink and Bouma (2013, Proposition 3), but we cannot
} 
Solving for $t$ and substituting $\lambda(m) / m$ for $\kappa(m)$, we combine both conditions in a chained inequality:

$$
m \lambda(m-1)-\lambda(m) \leq t<(m+1) \lambda(m)-\lambda(m+1) .
$$

Substituting $\lambda$ using (1) in the LHS and RHS of (7) and subsequent rearranging we find:

$$
\frac{1}{(n-m+1)^{2}}+\frac{m}{(n-m+3)^{2}}<\frac{m+2}{(n-m+2)^{2}}
$$

which holds for all combinations $(m, n)$ except for the grand coalition $(m=n)$ when $n \leq 3.5$ Note that these exceptions are trivial cases since $m=n$ implies $s=0$ and so there is no supporter to pay the transfer. Hence, for all non-trivial combinations $(m, n)$ there exist supporting transfers that can internally and externally stabilize coalitions of size $m$.

Uniqueness is verified by checking any overlap in the two conditions for coalitions of different size. Note that the LHS of (7) for an $m+1$ size coalition, $(m+1) \lambda(m)-$ $\lambda(m+1)$, is equivalent to the RHS of (7) for a coalition of size $m$. This equivalence implies that condition (7) is violated (notice the strict inequality sign), such that only coalitions of one size are both internally and externally stable. Hence, by selecting their transfer $t(s)$, the set of supporters $s$ is implicitly selecting a unique coalition size $m$, confirming our claim on uniqueness made in Section 2.2 .

Two questions remain. One is, in case of multiple possible transfers $t(s)$ and related coalition sizes $m(t(s)$ ), which of these maximizes supporters' payoffs $\lambda(m)-$ $t(s) / s$. This question corresponds to the stage 2 decision by the supporters. The other question is whether in equilibrium there exist supporters willing to pay such transfers. This question requires the analysis of the stage 1 stability conditions.

In stage 2, we employ (5) to determine the supporters' selection of transfers. Transfers are made to internally stabilize a coalition of size $m$ without wasting money,

use their proof to check these conditions since it contains a minor error. In Equation (A16) of Ansink and Bouma (2013), the denominator of the first term should have $s$ replaced by $(s+1)$, since the coalition payoff is shared by $s+1$ members if player $j$ joins. Hence, our analysis is slightly different.

${ }^{5}$ Substituting $m=n-x$ with $x \geq 0$ and solving for $n$, the inequality in (8) can be written as $n>\left(x^{4}+3 x^{3}+5 x^{2}+17 x+18\right) /\left(2 x^{3}+9 x^{2}+12 x+5\right)$. Since we require $m \geq 1$, the minimum number of players given $x$ equals $n \geq x+1$. We substitute $n=x+1$ and solve for $x$ to find that the inequality holds for any integer $x \geq 1$. For the trivial case $x=0 \Leftrightarrow m=n$ we obtain $1+\frac{n}{9}<\frac{n+2}{4}$, which holds for any integer $n \geq 4$, confirming the two exceptions presented in the main text. 
so we use (3) to write the transfer needed to stabilize a coalition of size $m: 6$

$$
t=m \lambda(m-1)-\lambda(m)
$$

Since we know that each transfer induces a unique equilibrium coalition size, we rewrite the supporters' maximization problem in (5) as a problem of the choice of $m$. Substituting $t$ using (9) we have:

$$
m(t(s))=\arg \max _{m^{\prime} \leq n-s}\left((s+1) \lambda\left(m^{\prime}\right)-m^{\prime} \lambda\left(m^{\prime}-1\right)\right) .
$$

The domain of $m$ is restricted since we need supporters to provide support, hence $m \leq n-s$.

We write out the Lagrangian with $\gamma$ denoting the Lagrange multiplier:

$$
\mathscr{L}=((s+1) \lambda(m)-m \lambda(m-1))-\gamma(m-n+s) .
$$

The related first order condition and complementary slackness condition to be solved are:

$$
\begin{aligned}
& 0=\frac{\partial \mathscr{L}}{\partial m}=\frac{2(s+1)}{(n-m+2)^{3}}-\frac{n+m+3}{(n-m+3)^{3}}-\gamma, \\
& 0=\gamma(m-n+s) .
\end{aligned}
$$

Interior solutions Solutions with $\gamma=0$ give the interior case where $m \leq n-s$. Rearranging (12) we obtain

$$
s=\frac{(n+m+3)(n-m+2)^{3}}{2(n-m+3)^{3}}-1 .
$$

Polynomial division of (14) yields an upward sloping asymptote $s=\frac{n+m-2}{2}$, with a remaining fraction that tends to 0 from below as $n$ increases. We proceed to check limit behavior as $n$ becomes large, while results for small $n$ will be assessed numerically below. For sufficiently large $n$, this asymptote implies that a 1-player change in the number of supporters induces a 2-player change in the number of

\footnotetext{
${ }^{6}$ For the trivial case $m=1$ we have $m \lambda(m-1)-\lambda(m)<0$, and hence no support will be paid. In this case we set $t=0$.
} 
coalition members, 7

We proceed to stage 1 and substitute this relation between $s$ and $m$ in stability conditions (6b) and (6d) (note that it is sufficient to show the violation of just one of the four stability conditions in this stage). We also substitute $t(s)$ using (9) and rearrange terms to obtain:

$$
\begin{aligned}
& \lambda(m-1) \leq\left(\frac{n+m}{2 m}\right) \lambda(m)-\left(\frac{n+m-2}{2 m}\right) \lambda(m-2), \\
& \lambda(m-1) \geq\left(\frac{n+m+2}{n+m}\right) \lambda(m+2)-\left(\frac{2 m+4}{n+m}\right) \lambda(m+1) .
\end{aligned}
$$

We will show that there do not exist interior solutions with support that satisfy both conditions. Combining (15) and (16) in a chained inequality gives the necessary condition

$$
\begin{aligned}
\left(\frac{n+m+2}{n+m}\right) \lambda(m+2)-\left(\frac{2 m+4}{n+m}\right) \lambda(m+1) & \\
\leq & \lambda(m-1) \leq\left(\frac{n+m}{2 m}\right) \lambda(m)-\left(\frac{n+m-2}{2 m}\right) \lambda(m-2) .
\end{aligned}
$$

Next, we verify the existence of at least one combination of integers $(m, n)$ such that (17) holds. We do so on the domain $m \leq n-s$. Using the asymptote $s=\frac{n+m-2}{2}$, hence assuming large $n$, this domain can be written as $n \geq 3 m-2$. On this domain, we find numerically that condition (17) is violated for any combination $(m, n)$ with two exceptions: $(m=2 \wedge n \geq 7)$ and $(m=3 \wedge n \geq 10)$. These particular parameter combinations, however, violate either condition (6b) or (6d). Hence, for this interior case with large $n$, no equilibria with support exist.

We proceed to assess the results of stages 1 and 2 for the interior case and small $n$. We do so numerically. Since the above-described limit behavior occurs already for $n \geq 23$, we check all cases where $n<23$. Doing so, we do not find any equilibria with support.

Boundary solutions Solutions with $\gamma \neq 0$ give the boundary case where $m=n-s$, so we have no free-riders. We will see that this boundary case gives rise to two equilibrium solutions with support. To show this we proceed to stage 1 and assess

\footnotetext{
${ }^{7}$ In approaching the asymptote, there are occasional 3-player changes in the number of coalition members. This case is approached similarly to 2-player changes and yields similar results. For brevity, it is not reported here.
} 
stability condition (6a) or (6b), depending on which case materializes, as discussed below (again, it is sufficient to show the violation of one of the four stability conditions in this stage). Different cases may arise since at the boundary we are not sure how the number of members changes in stage 3 in response to a supporter deviating in stage 1. This change in members is positive if the Lagrange multiplier is sufficiently high, inducing another boundary solution with $s-1$ supporters and $m+1$ members. This change in members is (weakly) negative, however, if the situation with one supporter less induces an interior solution. From the asymptotic behavior described for the interior case we know that a reduction in the number of members in response to a deviating supporter can never go beyond -3 members (see Footnote 7). Hence, we have $m(t(s-1))=m(t(s))+x$ with $x \in\{-3,-2,-1,0,1\}$.

For $x=1$, condition (6b) does not apply and we focus on condition (6a). Using (9) to simplify this condition and substituting $m(t(s-1))=m(t(s))+1$ we obtain

$$
\lambda(m(t(s)))-\frac{t(s)}{s} \geq \lambda(m(t(s)))
$$

Since $\lambda(m)$ is increasing in its argument, as can be seen from (1), this condition is violated for any $m=n-s$.

For $x \in\{-3,-2,-1,0\}$, we focus on condition (6b), which is stronger than (6a). Starting with $x=0$, we find a closed-form solution for the zero of condition (6b), which dictates that condition (6b) is violated for all $m \geq 2$ :

$$
\begin{aligned}
m & =\left(\frac{\sqrt{-4 n^{3}-16 n^{2}+16 n+87}}{23^{\frac{3}{2}}}-\frac{2 n^{3}+6 n^{2}-12 n-43}{54}\right)^{\frac{1}{3}}-\frac{-2 n-5}{3} \\
& +\frac{n^{2}+2 n-5}{9}\left(\frac{\sqrt{-4 n^{3}-16 n^{2}+16 n+87}}{23^{\frac{3}{2}}}-\frac{2 n^{3}+6 n^{2}-12 n-43}{54}\right)^{-\frac{1}{3}} .
\end{aligned}
$$

Repeating this analysis for the other values of $x$ shows that condition (6b) is violated for

$$
\begin{cases}m \geq 2 & \text { if } x=0, \\ m \geq 3 & \text { if } x=-1 \\ \{m \geq 5, m=4 \wedge n \leq 10\} & \text { if } x=-2 \\ \{m \geq 7, m=4 \wedge n \leq 6, m=5 \wedge n \leq 11, m=6 \wedge n \leq 25\} & \text { if } x=-3\end{cases}
$$


Combining the above cases, a sufficient condition for violation of condition (6b) is that the number of members is sufficiently large: $m \geq 7$.

We proceed to assess the results of stages 1 and 2 numerically for the interior case and $m<7$. Doing so, we find two equilibria with support:

$$
\left(s^{*}, m^{*}, f^{*}\right) \in\{(1,2,0),(2,3,0)\}
$$

No other equilibrium with support exists and this result represents only a marginal improvement on the case without supporters where the only non-trivial equilibrium is $m^{*}=2$ if $n=2$ (Pintassilgo and Lindroos 2008).

Surprisingly, although we find a support equilibrium for both $n=3$ and $n=5$, we do not find one for $n=4$. Going from 3 to 4 players, the additional player undermines coalition stability irrespective of his player type. If he were a free-rider such that $(s, m, f)=(1,2,1)$, he would undermine external stability of the set of supporters; condition (6d) is violated. If he were a member such that $(s, m, f)=(1,3,0)$, the single supporter is not willing to provide support to the resulting 3-player coalition; applying (10) for $s=1$ does not result in $m=3$. Finally, if he were a supporter such that $(s, m, f)=(2,2,0)$, he cannot increase the size of the coalition so he has an incentive to deviate; condition (6b) is violated.

\section{Illustration}

In this section we illustrate our main result with an example. This example highlights the players' choices in stage 1 and stage 3 of the game and shows how these choices depend on the number of players and their partitioning in player types $(s, m, f)$. Players' choices are guided by comparisons of equilibrium payoffs (1) and (2) as well as transfers (9). These functions depend partly on bio-economic model parameters $r, p, k$, and $\beta \equiv \frac{c}{p q k}$. In the simulations we choose to keep these bio-economic parameters fixed and vary only $n$ and its partitioning $(s, m, f)$. In choosing the bio-economic parameters, keep in mind that these only scale equilibrium payoffs and as such, their selection is not crucial for the illustration. We take parameters from a pelagic fishery as used by Seijo et al. (1998), see Table 1. Jointly, these parameter values imply that $\beta \equiv \frac{c}{p q k} \approx 0.36$ so that the numerator of (1) and (2) equals $\operatorname{rpk}(1-\beta)^{2} \approx 31 \cdot 10^{6}$. 
Table 1: Bio-economic parameters.

\begin{tabular}{lcc}
\hline Intrinsic growth rate & $\mathrm{r}$ & 0.36 \\
Carrying capacity & $\mathrm{k}$ & 3500000 \\
Catchability coefficient & $\mathrm{q}$ & 0.0004 \\
Price & $\mathrm{p}$ & 60 \\
Marginal effort cost & $\mathrm{c}$ & 30000 \\
\hline
\end{tabular}

In stage 3 , the question is how many singletons will join the coalition. Figure 1 illustrates, for selected values of $n \geq 3$, which stage 3 coalition size $m(s(t))$ is implicitly chosen by the set of supporters $s$ through the transfer $t(s)$ in stage 2 . Each of the marks depicts the unique internally and externally stable coalition size $m$ that maximizes the supporters' payoffs. Given $n$, we observe for small $s$ that $m(t(s))$ is weakly increasing, illustrating that larger groups of supporters choose to support larger coalitions, but only if there is a sufficiently large set of supporters. The increasing slope is not linear due to the discrete nature of our game. Once the constraint $m \leq n-s$ kicks in, boundary solutions imply that the line connecting the $m(t(s))$ marks starts to decrease with slope -1 .

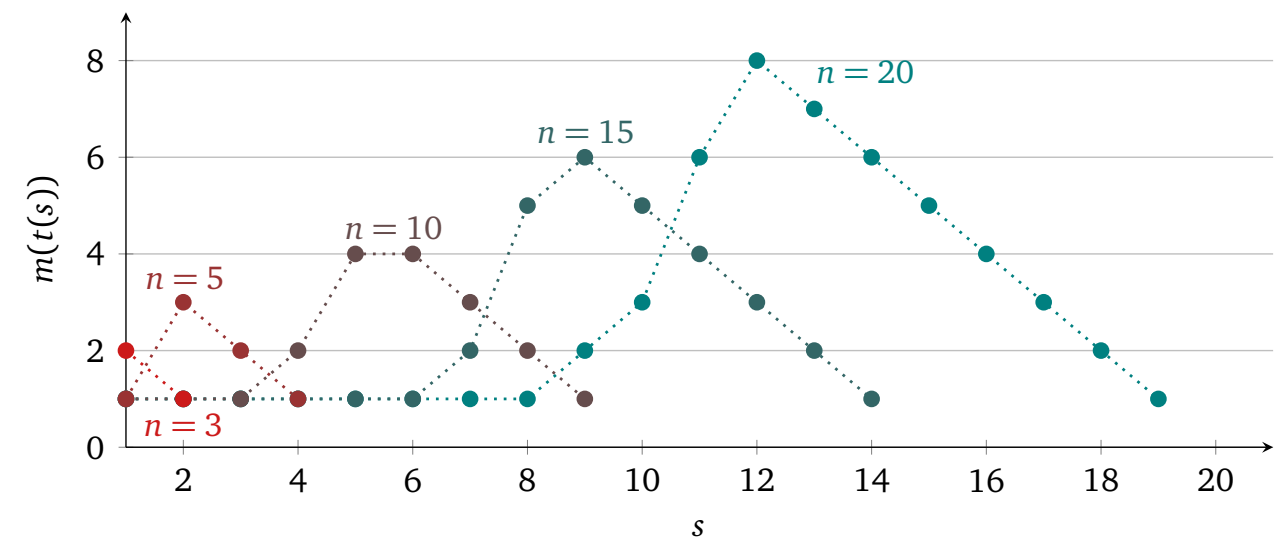

Figure 1: Implicit choice of $m(t(s))$ by the supporters in stage 3 as a function of $s \leq$ $n-1$ for selected values of $n$.

In stage 1, the question is how many players will choose to become a supporter. These choices are driven by payoff comparisons, taking into account subsequent decisions in the remaining stages of the sequential game. Figure 2 illustrates payoffs to each of the three player types for two observations from Figure 1 : $n=5$ and $n=10$. In both panels, for each $s$ and related $m(t(s)$ ) (as shown in Figure 1), we observe free-rider payoffs $\lambda(m(t(s)))$, supporter payoffs $\lambda(m(t(s)))-t(s) / s$ and member 
payoffs $\kappa(m(t(s)))+t(s) / m(t(s))$. Several features of this figure are noteworthy. First, payoffs to each player type at $s=1$ and $s=n-1$ are identical: these solutions correspond to the trivial case where each player behaves as a singleton. Second, for each player type, payoffs are hump-shaped in $s$, peaking at the point where the constraint $m \leq n-s$ kicks in, implying that all players are better off with fewfree riders and a large coalition. Third, sharp increases in member and free-rider payoffs occur when a change in $s$ induces a large jump in the number of members: $m(t(s))-m(t(s-1))>1$. One such example is in the right panel when $s$ increases from $s=4$ to $s=5$, which induces a jump of $m(t(5))-m(t(4))=2$ as is shown in Figure 1. Fourth, member payoffs stay close to free-rider payoffs due to internallystabilizing transfers; since these transfers are paid by the supporters, their payoff is generally low.
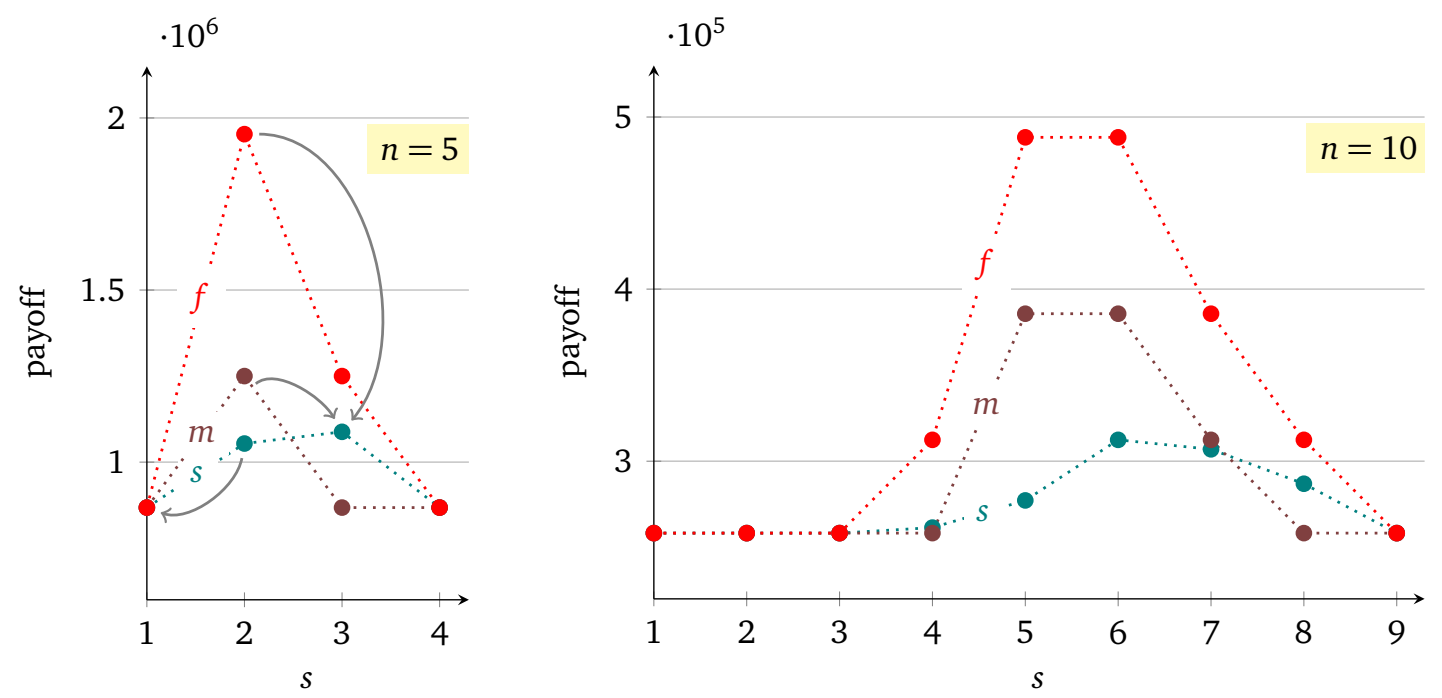

Figure 2: Payoffs (including transfers) to supporters $s$, members $m$ and free-riders $f$ as a function of $s \leq n-1$ for two values of $n$ (left panel: $n=5$, right panel: $n=10$ ), taking into account $m(t(s))$ as illustrated by Figure 1. Note the difference in y-axis scales. Equilibrium solution $\left(s^{*}, m^{*}, f^{*}\right)=(2,3,0)$ is visible in the left panel; gray arrows illustrate that stage 1 deviations from this solution give lower payoffs.

Our main result is illustrated in the left panel of Figure 2, Recall that one of the two equilibrium solutions that we found in Section 3 equals $\left(s^{*}, m^{*}, f^{*}\right)=(2,3,0)$ which occurs for $n=5$. The gray arrows in the left panel of Figure 2 depict changes in payoffs upon deviation for this solution. The arrows correspond to stability conditions (6a)- $-6 \mathrm{~d}$ ). Since each of the arrows points downward (note that the arrows 
for (6a) and (6b) overlap), the figure confirms that all stability conditions hold and $\left(s^{*}, m^{*}, f^{*}\right)=(2,3,0)$ is an equilibrium solution. Visual inspection reveals that none of the other solutions $(m, s, f)$ depicted in both panels of Figure 2 shares this feature, which confirms the result found in Section 3 .

\section{Conclusion}

Given the bleak results on coalition formation in CPR games, in this paper we explored the prospect of support in such games. We find that support yields an additional equilibrium when there are three or five players. No equilibria with support exist for games with more than five players which confirms that cooperation is particularly hard in larger CPR games, when one does not consider aspects like communication, sanctioning, reputation or norms (cf. Ostrom 1990). We conclude with descriptions of two model alternatives and their impacts on results.

The first alternative is restricting the supporters' choice of transfers in stage 2 of the game. Our paper is closely related to Ansink et al. (2018), who studied a public goods game with support. For two common specifications of this game, they did not find any equilibrium with support and resorted to introducing an agency that designs a transfer scheme which the supporters can either reject or accept in stage 2 of the game. Their existence result for equilibria with support in this restricted version of the game depends on condition (6b) as well as the assumption that $\lambda(m)$ is increasing in its argument. Since our $\lambda(m)$ satisfies this property, their existence result can be applied directly to our setting. Introducing an agency that sets the transfer scheme would result in multiple equilibria with support $\left(s^{*}, m^{*}, f^{*}\right)$ for every $n$, including some with large $m^{*}$. An important reservation about this positive result is of course the pertinence of introducing an agency for the specific CPR problem at hand.

The second alternative is to consider the Stackelberg version of the CPR game assessed here. In this game, the coalition chooses its stage 4 effort level before the singletons do. The Stackelberg game leads to the alternative payoff functions $\lambda(m)=r(1-\beta)^{2} / 4(n-m+1)^{2}$ and $\kappa(m)=r(1-\beta)^{2} / 4 m(n-m+1)$ (Long and Flaaten 2011). Without support, this game already leads to large coalitions with about half the number of players. Using a similar analysis as for the Cournot case assessed above, we find that adding support does not yield any additional equilibria. We conclude that support has little impact on the outcomes of CPR games. 


\section{References}

Ansink, E., H.-P. Weikard, and C. Withagen. 2018. "International environmental agreements with support". Forthcoming in Journal of Environmental Economics and Management.

Ansink, Erik, and Jetske Bouma. 2013. "Effective support for community resource management". Forest Policy and Economics 1 (37): 94-103.

Barrett, S. 1994. "Self-enforcing international environmental agreements". Oxford Economic Papers 46 (S): 878-894.

Carraro, C., and D. Siniscalco. 1993. "Strategies for the international protection of the environment". Journal of Public Economics 52 (3): 309-328.

Clark, C.W. 1990. Mathematical Bioeconomics, 2nd Ed. New York: Wiley.

D’Aspremont, C., et al. 1983. "On the stability of collusive price leadership". Canadian Journal of Economics 16 (1): 17-25.

Doyen, L., and J.C. Péreau. 2012. "Sustainable coalitions in the commons". Mathematical Social Sciences 63 (1): 57-64.

Gordon, H.S. 1954. "The economic theory of a common-property resource: The fishery". Journal of Political Economy 62 (2): 124-142.

Heintzelman, M., S. Salant, and S. Schott. 2009. "Putting free-riding to work: A partnership solution to the common-property problem". Journal of Environmental Economics and Management 57 (3): 309-320.

Hoel, M. 1992. "International environment conventions: The case of uniform reductions of emissions". Environmental and Resource Economics 2 (2): 141-159.

Johannesen, A.B., and A. Skonhoft. 2009. "Local common property exploitation with rewards". Land Economics 85 (4): 637-654.

Kwon, O. 2006. "Partial international coordination in the great fish war". Environmental and Resource Economics 33 (4): 463-483.

Long, L., and O. Flaaten. 2011. "A Stackelberg analysis of the potential for cooperation in straddling stock fisheries". Marine Resource Economics 26 (2): 119-139.

Mesterton-Gibbons, M. 1993. "Game-theoretic resource modeling". Natural Resource Modeling 7 (2): 93-147. 
Ostrom, E. 1990. Governing the Commons: The Evolution of Institutions for Collective Action. Cambridge: Cambridge University Press.

Pintassilgo, P., and M. Lindroos. 2008. "Coalition formation in straddling stock fisheries: A partition function approach". International Game Theory Review 10 (3): 303-317.

Pintassilgo, P., L. Kronbak, and M. Lindroos. 2015. "International fisheries agreements: A game theoretical approach". Environmental and Resource Economics 62 (4): 689709.

Polasky, S., et al. 2006. "Cooperation in the commons". Economic Theory 29 (1): 71-88.

Schaefer, M. 1954. "Some aspects of the dynamics of populations important to the management of the commercial marine fisheries". Inter-American Tropical Tuna Commission Bulletin 1 (2): 23-56.

Seijo, J.-C., O. Defeo, and S. Salas. 1998. "Fisheries bioeconomics: theory, modelling and management". FAO Fisheries Technical Paper 368.

Weikard, H.-P. 2009. "Cartel stability under an optimal sharing rule". The Manchester School 77 (5): 575-593.

Zavalloni, M., M. Raggi, and D. Viaggi. 2018. "Agri-environmental policies and public goods: An assessment of coalition incentives and minimum participation rules". Forthcoming in Environmental and Resource Economics. 


\section{Appendix: Equilibrium payoffs of the CPR game}

The game for which we examine the potential impact of support is the canonical version of a CPR game (cf. Mesterton-Gibbons 1993), which is based on the classical Gordon-Schaefer bio-economic model (Gordon 1954; Schaefer 1954). It features a linear harvest function and a logistic growth function. We denote a player's individual harvest by

$$
h_{i} \equiv q e_{i} X
$$

where $q$ is the so-called catchability coefficient, $e_{i}$ is individual effort and $X$ is the resource stock. The stock grows with intrinsic growth rate $r$ according to a logistic growth function

$$
\frac{d X}{d t}=r X\left(1-\frac{X}{k}\right)
$$

where $k$ is the system's carrying capacity.

Payoff functions are derived for the system's steady state, i.e. where harvest equals growth such that $\sum_{i \in N} q e_{i} X=r X\left(1-\frac{X}{k}\right)$. In the steady state we have

$$
\sum_{i} q e_{i} X=r X\left(1-\frac{X}{k}\right)
$$

Solving for $X$ we obtain the steady state stock:

$$
X=\frac{k}{r}\left(r-q \sum e_{i}\right)
$$

Individual payoffs in the steady state are given by $v_{i}=p h_{i}-c e_{i}$ (Clark 1990), where $p$ is the price for selling one's harvest and $c$ is the marginal effort cost, assuming that an individual's share of the harvest is equal to his share of efforts: $h_{i} / \sum_{j} h_{j}=e_{i} / \sum_{j} e_{j}$. Player i's best response function is obtained by solving

$$
\max _{e_{i}}\left(p\left(\frac{e_{i}}{\sum_{j} e_{j}}\right) \sum_{j} h_{j}-c e_{i}\right) .
$$


Define $e_{-i} \equiv \sum_{j} e_{j}-e_{i}$, then the first order condition gives

$$
\frac{c}{p q k}=1-\left(\frac{q}{r}\right) e_{-i}
$$

Define $\beta \equiv \frac{c}{p q k}$, then we can write the best response function as

$$
e_{i}^{*}=\frac{r(1-\beta)}{2 q}-\left(\frac{1}{2}\right) e_{-i}
$$

We make two simplifications. First, for symmetric players we have $e_{-i}=(n-1) e_{i}$. Second, since the coalition behaves like a single player, if $m$ players form a coalition, we can effectively model a game with $n-m+1$ players harvesting the resource. The coalition's and each singleton player's best response function can now be written and solved for $e_{i}^{*}$ to obtain the equilibrium effort level:

$$
e_{i}^{*}=\frac{r(1-\beta)}{q(n-m+2)} \text {. }
$$

Note that the coalition's effort level, and hence its share of the harvest is the same as the effort level of each individual singleton. Substituting equilibrium effort levels into the individual payoff function, we obtain equilibrium payoffs to singletons and the coalition:

$$
v_{M}(m)=v_{i}(m)=\frac{r p k(1-\beta)^{2}}{(n-m+2)^{2}}, i \in N \backslash M,
$$

where the numerator consists of bio-economic model parameters only (cf. Pintassilgo et al. 2015). A coalition member must share this payoff with the other members and earns only a fraction $\frac{1}{m}$ of what a singleton earns. 